\title{
A human gut phage catalog correlates the gut phageome with type 2 diabetes
}

Yingfei Ma ${ }^{*}$, Xiaoyan You, Guoqin Mai, Taku Tokuyasu and Chenli Liu*

\begin{abstract}
Background: Substantial efforts have been made to link the gut bacterial community to many complex human diseases. Nevertheless, the gut phages are often neglected.

Results: In this study, we used multiple bioinformatic methods to catalog gut phages from whole-community metagenomic sequencing data of fecal samples collected from both type II diabetes (T2D) patients $(n=71)$ and normal Chinese adults $(n=74)$. The definition of phage operational taxonomic units (pOTUs) and identification of large phage scaffolds ( $n=2567, \geq 10 \mathrm{k}$ ) revealed a comprehensive human gut phageome with a substantial number of novel sequences encoding genes that were unrelated to those in known phages. Interestingly, we observed a significant increase in the number of gut phages in the T2D group and, in particular, identified 7 pOTUs specific to T2D. This finding was further validated in an independent dataset of 116 T2D and 109 control samples. Co-occurrence/exclusion analysis of the bacterial genera and pOTUs identified a complex core interaction between bacteria and phages in the human gut ecosystem, suggesting that the significant alterations of the gut phageome cannot be explained simply by co-variation with the altered bacterial hosts.

Conclusions: Alterations in the gut bacterial community have been linked to the chronic disease T2D, but the role of gut phages therein is not well understood. This is the first study to identify a T2D-specific gut phageome, indicating the existence of other mechanisms that might govern the gut phageome in T2D patients. These findings suggest the importance of the phageome in T2D risk, which warrants further investigation.
\end{abstract}

Keywords: Phageome, Metagenomics, T2D, Alterations, Correlation

\section{Background}

The gut microbiota has increasingly been recognized as a key contributor to human health, and various chronic human diseases can be linked to dysbiosis of the intestinal microbiota [1-7]. The human gut virome, also known as the phageome, refers to the whole community of viruses in the gut, most of which are bacteriophages. Bacteriophages are thought to be the most abundant biological entities in the human gut ecosystem. There may be at least $10^{9}$ viruslike particles (VLPs) per gram of human feces, ten times more than that of bacterial cells $[8,9]$. However, phageome studies remain challenging because of the extremely high diversity of the phage community, highly divergent phage

\footnotetext{
* Correspondence: yingfei.ma@siat.ac.cn; cl.liu@siat.ac.cn Institute of Synthetic Biology, Shenzhen Institutes of Advanced Technology, Chinese Academy of Sciences, 1068 Xueyuan Avenue, University Town, Nanshan, Shenzhen 518055, China
}

genomes, and lack of a universal marker akin to the bacterial 16S rRNA genes.

Recent advances in next-generation sequencing (NGS) technology and bioinformatic tools have fostered the development of large-scale studies of the human gut phageome $[7,9]$. One approach based on metagenomic analysis of VLPs focuses primarily on the free phages at the time of sampling [10-14]. In whole-community metagenomic sequencing (WCMS)-based approaches, it is estimated that up to $17 \%$ of the WCMS-identified DNA sequences from stool samples are of phage origin $[10,15]$. WCMS data that contain valuable information about phages, prophages, and their bacterial hosts can be used to predict the interactions between gut phages and their bacterial hosts, allowing indepth study of the dynamics of gut microbial communities (for example, see refs. [7, 9, 16-18]).

Bacteriophages play roles in intestinal physiology that are far more important than the alteration of bacterial 
communities by phage infection [19-25]. For instance, phages residing in mucosal surfaces can provide nonhost-derived immunity against bacterial infections [26]. A very recent study uncovered inflammatory bowel disease (IBD)-specific alterations in the enteric virome [14, 27]. Therefore, the gut phageome may play important roles in T2D, obesity, and related diseases.

Qin et al. found that T2D is linked to gut bacterial dysbiosis using a metagenomic analysis of WCMS data [3] but neglected the role of gut phages in T2D. In this study, utilizing a WCMS dataset developed from fecal samples from a total of 370 Chinese T2D patients and nondiabetic controls [3], we used three bioinformatic strategies to identify large scaffolds of phage origin and defined phage operational taxonomic units (pOTUs) to obtain insights into the human gut phageome. This is the first study to correlate the gut phageome with $\mathrm{T} 2 \mathrm{D}$, and further investigations are required to determine if the gut phageome contributes to T2D risk.

\section{Methods}

\section{Metagenomic datasets used in this study}

All WCMS reads used in this study were obtained from the NCBI short read archive (SRA) database (SRA045646) generated from a previously published Chinese T2D microbiome study [3]. The study was a case-control study that included 187 cases and 183 controls. Similar to the published study, we divided our study into two stages. Stage I was an exploration stage (control $n=74, \mathrm{~T} 2 \mathrm{D} n=71$ ), and stage II was a validation stage (control $n=109$, T2D $n=$ 116). Additionally, the corresponding assembled scaffolds generated from the exploration stage were downloaded from GigaDB (gigadb.org/dataset/100036) [3].

The VLP-based metagenomic sequencing datasets generated in three previous studies were downloaded from the EMBL-EBI (PRJEB7772) and NCBI SRA (SRP021107, SRX020505, and SRX020504) [10, 12, 14]. These data of phage origin were used to capture new phages in the WCMS data and to compare the differences in phage distribution between the WCMS data and VLP-based sequencing data.

All datasets used in this study are listed in Additional file 1: Table S1.

\section{Detection of known phage genomes in the metagenome} Known bacterial phage genomes were downloaded from the European Nucleotide Archive database (ENA, http:// www.ebi.ac.uk/genomes/phage.html) and formatted as the ENA phage database (ENADB), and the corresponding protein sequences were formatted as the European Nucleotide Archive Protein Database (ENAPDB). Overall, the ENADB contains 2501 phage genomes associated with 2010 bacterial host species. The WCMS reads (145 samples in stage I of the T2D study) and VLP reads (including all three VLP metagenomic datasets (Additional file 1: Table S1)) were mapped to the genomes in the ENADB by the software Soap2. The breadth of coverage and depth of coverage of each phage genome by the reads in each sample were estimated with default flags $[3,10,12,14,28]$. Only the ENA genomes with $>30 \%$ breadth of coverage were selected for display.

\section{Identification of large phage scaffolds ( $\geq 10 \mathrm{~kb}$ )}

Three strategies were developed to identify large scaffolds $(\geq 10 \mathrm{~kb})$ of putative phage origin in the assembled scaffolds of the T2D study (gigadb.org/dataset/100036) (Fig. 1a; see also Additional file 1: Materials and Methods for details). Briefly, we identified a large scaffold ( $\geq 10 \mathrm{~kb})$ as a candidate sequence of phage origin if (1) the scaffold was probed by spacer(s) of CRISPRs, (2) the scaffold was mapped by VLP metagenomic reads with a high breadth of coverage $(\geq 40 \%)$, and (3) the scaffold encoded gene(s) homologous to the gene(s) from ENA phages. Finally, all assigned scaffolds were manually checked, and the scaffolds with ambiguous assignments were discarded in the next analysis steps. Because most of the identified phage scaffolds in this study are part of phage genomes, we did not intend to distinguish between phage and prophage sequences. This process identified 2567 putative large phage scaffolds from the 145 stage I samples.

The relative abundance of each identified phage scaffold was estimated. To this end, we firstly mapped all reads to the identified phage scaffolds and counted the numbers of the reads mapping to the identified phage scaffolds in each sample, respectively; secondly, the read number corresponding to each identified phage scaffold was normalized by the total read number of each sample; the normalized value thereby represents the relative abundance of each phage scaffold in each sample.

\section{Definition of phage operational taxonomic units and profile of bacterial genera}

An operational taxonomic unit (OTU) is defined as a group of closely related individuals that share a given set of observed characters or undetermined evolutionary relationships [29]. We bioinformatically defined phage OTUs (pOTUs) (Fig. 2b; see also Additional file 1: Materials and Methods for details) to profile the phageome and examine the interactions between phages and bacteria in the gut ecosystem. Briefly, we constructed an Expanded PhageSpecific Gene database (EPSGDB) according to the Phage Orthologous Groups (POGs) and the phage classification defined by the International Committee on Taxonomy of Viruses (ICTV) [17, 30]. A pOTU was defined as the collection of all phages with the same taxonomic names at all five levels (group|order|family|subfamily|genus) and sharing hosts within the same genera and a pOTU is not necessary to be associated with species or genus. Thus, a pOTU 

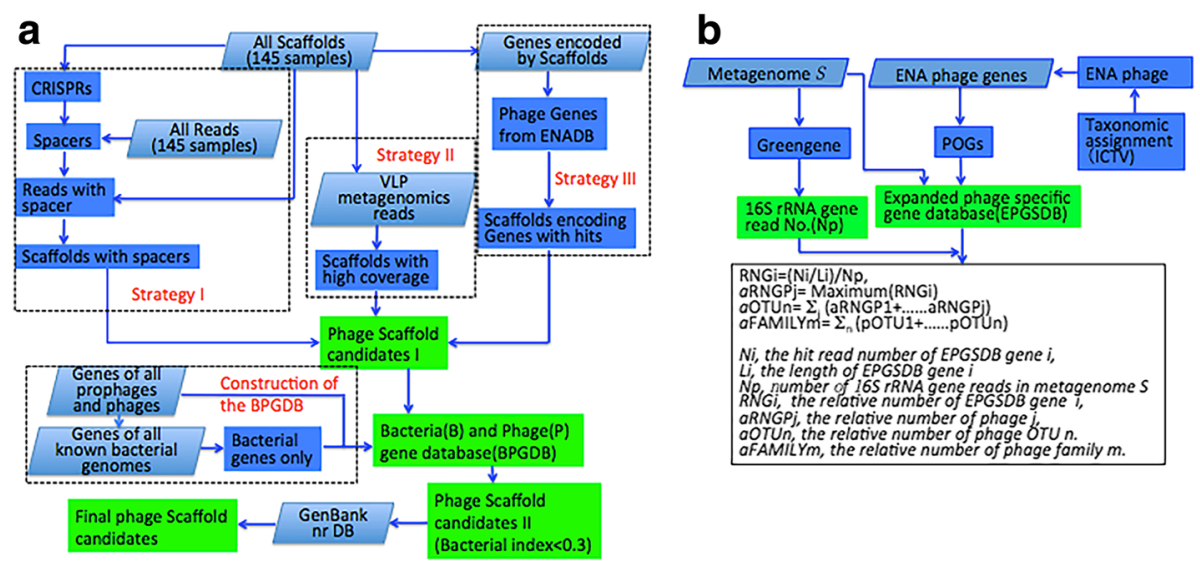

Fig. 1 Identification of the large phage scaffolds and definition of pOTU. a The pipeline used three strategies to identify the large phage scaffolds ( $\geq 10 \mathrm{k}$ ) (see also supplementary Materials and Methods for details). In strategy l, the scaffolds were probed by CRISPR spacer(s). In strategy II, the scaffolds were mapped by reads from the VLP metagenome with a high breadth of coverage $(\geq 40 \%)$. In strategy III, the scaffolds encoding genes homologous to those of ENA phages were identified. The Bacteria and Phage Gene Database (BPGDB) was constructed. The assigned scaffolds were manually checked by comparing them against the BPGDB and GenBank nr database. The scaffolds with ambiguous assignments were discarded in the next analysis steps. $\mathbf{b}$ Definition of pOTUs to profile the phageome on the read level (see also supplementary Materials and Methods for details). The Expanded Phage-Specific Gene database (EPSGDB) was constructed according to the Phage Orthologous Groups (POGs). The phage taxonomical classification was performed based on the International Committee on Taxonomy of Viruses (ICTV) [17, 30]. The relative number of a pOTU in a sample was calculated by summing the numbers of all phage genomes belonging to the POTU and dividing by the number of $16 \mathrm{~S}$ rRNA gene reads in the sample
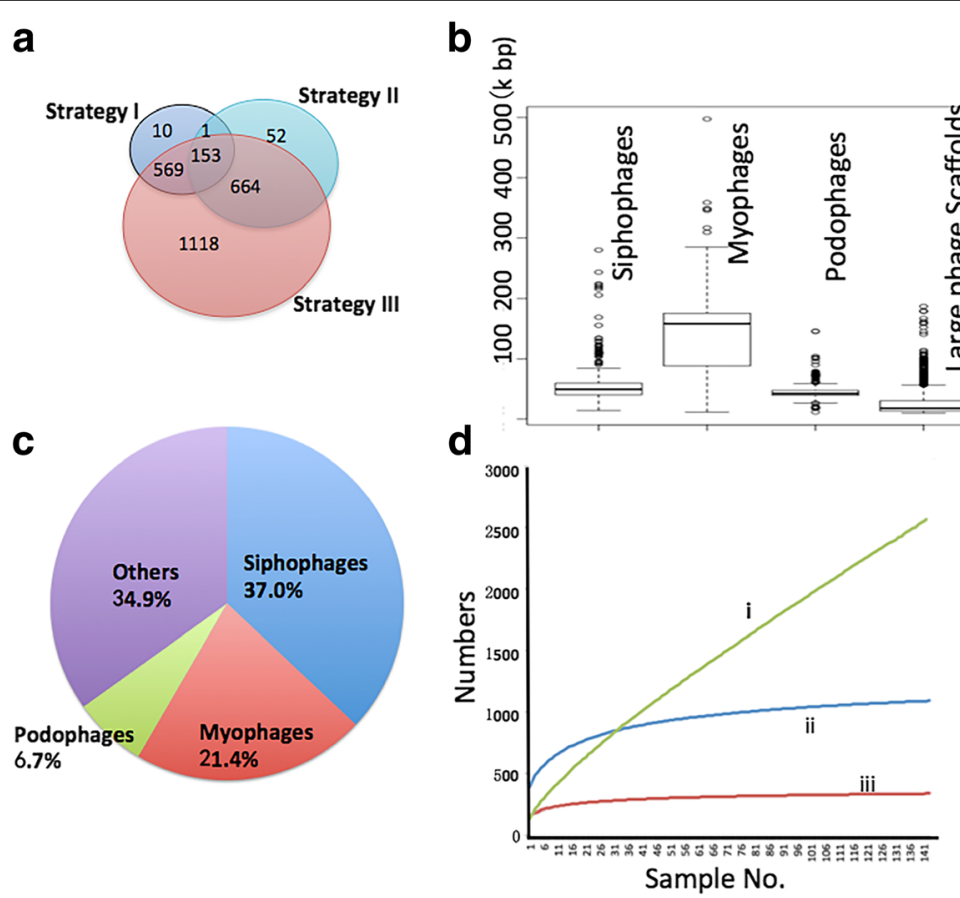

Fig. 2 Statistics of the identified phageome in the samples. a Venn diagram showing the numbers of the large phage scaffolds identified by the three strategies. $\mathbf{b}$ Length range of the genomes of siphophages, myophages, podophages, and the identified large phage scaffolds. c The percentage of phage families identified by the large phage scaffolds. $\mathbf{d}$ Rarefaction curves of the phageome, based on the number of the identified large phage scaffolds (i), the number of ENA genomes with a high breadth of coverage in the metagenomes (ii), and the number of defined pOTUs in the metagenomes (iii) 
was named with an ordered list of phage names at all the five levels plus the genus name of the bacterial host. In brief, we defined a pOTU to represent a group of phages with homologous phage taxon-specific genes and the same bacterial genus of their hosts. The relative abundance of a pOTU in a sample was calculated by summing the numbers of all phage genomes belonging to the pOTU and dividing by the number of $16 \mathrm{~S}$ rRNA gene reads in the sample.

The MetaPhlAn software was used to profile the bacterial genera for each sample based on metagenomic reads with default flags [31].

\section{Gene annotation of the putative phage scaffolds}

The genes on the putative large phage scaffolds were predicted by MetaGeneMark with default flags [32], followed by functional annotation performed by comparing the genes to various databases, including the GenBank nr database, COG (Clusters of Orthologous Groups) database [33], Tigr (The Institute for Genomic Research) Microbial Database [34], and Pfam database of conserved amino acid motifs [35], using RPSblast (Reversed Position Specific Blast) with an $e$-value cutoff of $1 \mathrm{e}-10$ [36]. Virulence genes were identified by comparing the genes against the VFDB (Virulence Factor Database) with an $e$-value cutoff of 1e-05 [37].

\section{Taxonomic assignment of the large phage scaffolds and} the bacterial scaffolds ( $\geq 10 \mathrm{~kb}$ )

We taxonomically assigned each putative large phage scaffold $(\geq 10 \mathrm{~kb})$ on the phage family level. All the genes encoded by the phage scaffolds were queried against the EPSPDB with an $e$-value cutoff of 1e-05. Only the genes encoded by phage scaffolds with hits in the EPSPDB and only the top two hits were taken into consideration for the taxonomic assignment. The phage family of each hit was determined using the taxonomic information provided by the EPSPDB. Each gene encoded by the phage scaffolds contributed to the scaffold taxonomic assignment based on its hits, with the top hit weighted as 1 and the second as 0.5 . The phage family ratio (PFR) of a phage scaffold was calculated by normalizing the summed scores of each phage family by the total scores of the scaffold. A scaffold was assigned to a phage family if the PFR was larger than $50 \%$. All scaffolds with PFRs of no more than 50\% were assigned as unclassified.

The taxonomy of the bacterial large scaffolds ( $\geq 10 \mathrm{~kb}$ ) was assigned on the genus level using an approach similar to that of the phage scaffold assignment with modifications. All the genes encoded by the bacterial scaffolds were queried against the NCBI nr database, and the bacterial genus ratio (BGR) was calculated by normalizing the summed scores of each bacterial genus by the total scores of each bacterial scaffold, with a BGR $\geq 70 \%$ as the threshold for genus assignment.

\section{Construction of phylogenetic trees of gut phages based on large subunit terminase sequences}

Large subunit terminase (LST) sequences were selected as the marker to build phylogenetic trees of gut phages. The protein sequences annotated as LST were extracted from all ENA phage sequences. Pfam domains were searched using the hmmsearch program in the HMMER3 package (e-value cutoff 1e-05), and four domains, including Terminase_1 (PF03354), Terminase_3 (PF04466), Terminase_6 (PF03237) and Terminase_GpA (PF05876), were found on the LST sequences [38]. Thereafter, all the protein sequences encoded by the large phage scaffolds were searched against the Pfam database, and the protein sequences with one of the four functional domains were included in the tree construction. The alignment was performed and maximum likelihood trees were constructed by the program MEGA with 1000 bootstraps [39]. The trees were visualized by the Figtree software (http://tree.bio.ed.ac.uk).

\section{Construction of the interaction network of the gut bacteria and phages}

The co-correlation/exclusion between the gut bacteria and phages were calculated based on the relative numbers of bOTUs and pOTUs by SparCC (with $p<0.01$ and correlation $>0.3$ ) [40]. Only the bacterial genera and pOTUs with a high frequency (detected in more than 72 samples $(\geq 50 \%)$ ) were considered. The network layout was calculated and visualized using a circular layout by the Cytoscape software [41]. Only edges with correlations greater than 0.3 and a $p$ value less than 0.01 were shown, and unconnected nodes were omitted.

\section{Statistical analyses and data visualization}

The richness of the phageome in the 145 samples of stage I was estimated by the $\mathrm{R}$ package Vegan based on the Chao2 richness estimator. Two rarefaction curves of the gut phageome in the control samples and T2D samples were generated. The linear discriminant analysis (LDA) scores of the variations of bacterial genera and pOTUs between control samples and T2D samples were calculated and visualized by LEfSe (LDA Effect Size) [42]. Based on the relative abundances of the highly prevalent pOTUs detected in more than 120 samples (70\% of 145 samples), the significance of the variations of the gut phages between T2D and control groups were assessed by the Mann-Whitney rank-sum test with FDR correction [43]. The significant variation of the relative abundances of the identified phage scaffolds between the T2D and control groups was assessed by the Mann-Whitney rank-sum test.

\section{Results}

The human gut harbors a complex phageome

First, we estimated the gut phageome by mapping the WCMS reads against the known phage genomes in the 
European Nucleotide Archives genome database (ENADB). In the 145 WCMS metagenomes used for the stage I analysis, a total of 461 phage genomes were mapped with at least one read, with an average of $67( \pm 26)$ per sample. A total of 438 phage genomes in the ENAPDB were mapped by at least one read of the VLP metagenomic data $[10,12$, 14]. As shown in the figure (Additional file 2: Figure S1), most double-stranded (ds) DNA phage genomes exhibited a low breadth of coverage $(<20 \%)$ in both the VLP and WCMS data. Only a small fraction of the metagenomic reads $(0.55 \%$ reads of the WCMS data and $0.12 \%$ of the VLP data) could be mapped to the ENADB genomes. Considering that up to $17 \%$ of sequences in the WCMS data and most of the VLP metagenomic reads may be of phage origin $[10,15]$, these results suggest that most of the phages in the human gut are novel and are not yet included in the European Nucleotide Archives protein database (ENAPDB).

We next defined pOTUs to profile the gut phageome (Fig. 1b). In total, in the 145 WCMS samples, 341 pOTUs were assigned, averaging $96.7( \pm 26)$ pOTUs per sample. A majority of the pOTUs $(87.8 \pm 5.6 \%)$ belonged to the order Caudovirales. Siphoviridae was the most abundant family, accounting for $55.3 \pm 9.8 \%$ of all identified phages, followed by Myoviridae $(21.7 \pm 9.9 \%)$ and Podoviridae (10.6 $\pm 8.4 \%$ ) (Additional file 2: Figure S2).

Finally, using three bioinformatic strategies, we identified large phage scaffolds from the assembled scaffolds of the WCMS data for the stage I analysis (Fig. 1a). A total of 2567 scaffolds larger than $10 \mathrm{~kb}$ were putatively assigned as phage in origin (Fig. 2a, Additional file 3: Table S2). The large phage scaffolds ranged from 10 to $187 \mathrm{~kb}$ in length, which is consistent with the length range of phage genomes in the order Caudovirales (Fig. 2b). We taxonomically assigned each large phage scaffold as described in the "Methods" section. In total, 1671 of the 2567 (65.1\%) large phage scaffolds were qualified to assign on the family level. As observed for the pOTUs, the phages of Siphoviridae produced the largest number of scaffolds (950, 37.0\%), followed by Myoviridae (549, $21.4 \%$ ) and Podoviridae (172, 6.7\%) (Fig. 2c).

We estimated the richness of the identified phageome in this study. As shown in Fig. 2d, the rarefaction curve of the phageome, based on the number of the identified large phage scaffolds, grows rapidly across the 145 samples, suggesting that the gut phages are highly individualized. Based on the number of ENA genomes with a high breadth of coverage in the metagenomes and the number of defined pOTUs in the metagenomes, the rarefaction curves reach a plateau within approximately 20 samples, indicating that the pOTUs that we defined in this study are sufficient to represent the gut phageome.

\section{Analyses of the large phage scaffolds identify novel phagetypes and vast genetic diversity in the gut phageome}

In the 145 WCMS samples, most of the large phage scaffolds identified were unique to each sample, with a few exceptions of phage scaffolds widely distributed among individuals (Additional file 2: Figure S3a and b). The phages with a high breadth of coverage and depth of coverage were considered to be active at the time of sampling or to be prophages on bacterial genomes of some abundant taxa, such as Bacteroides spp. The newly identified crAssphage was one of the most prevalent phages and was observed in $23.4 \%(n=34)$ of the samples, and the largest crAssphage scaffolds were 90$100 \mathrm{~kb}$ in length, in line with the length of their reported prototype genome [44].

To establish the phylogeny and estimate the diversity of the uncultured gut phages, we selected large subunit terminase (LST) as a marker to construct phylogenetic trees. The LST sequences identified in the ENADB phage genomes had four different Pfam functional domains, including Terminase_1, Terminase_3, Terminase_6, and Terminase_GpA. In total, 576 LSTs were identified on the 2567 large phage scaffolds, with 111 belonging to the Terminase_1 domain, 145 to Terminase_3, 275 to Terminase_6, and 45 to Terminase_GpA. Phylogenetic trees of gut phages were constructed according to these domains (Fig. 3 and Additional file 2: Figure S4). As shown on the trees, some clusters did not include any known ENADB phages, likely representing novel phagetypes. The LST phylogeny expanded the diversity of gut phages and defined new lineages. Interestingly, there was no clear link between the types of terminases and phage families, highlighting the potential genetic plasticity of gut phages. Possible horizontal gene transfer might have obscured some robust phylogenetic signals of gut phages.

The genes encoded by the phage scaffolds were annotated by querying the sequences against general databases, including Pfam, COG, TIGR, and Bactnog. In total, of the 83,957 genes predicted from the phage scaffolds, only 19.3\% generated hits in the Expanded Phage-Specific Gene database (EPSDB), $18.8 \%$ in COG (Additional file 2: Figure S5), $15.4 \%$ in Tigr, $27.2 \%$ in Pfam, and $29.7 \%$ in the Bactnog database. Comparatively, $60.4 \%$ of genes in the integrated gene catalog of gut metagenomes generated hits in the Bactnog database [45], suggesting that most identified phage gene sequences are novel.

The large phage scaffolds identified in this study provided an opportunity to gain insights into the accessory gene pool carried by gut phages. A ubiquitous gene found in the large phage scaffolds encoded an IgA protease (Additional file 3: Table S3), whose putative function is to allow the bacterial hosts or phages to adhere to mucous 


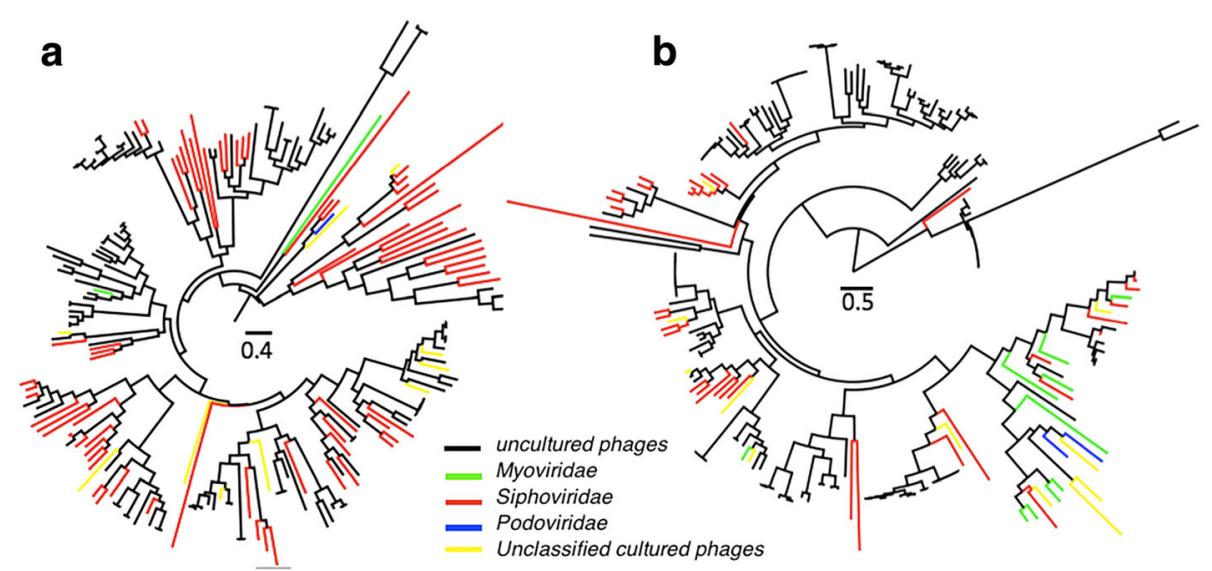

Fig. 3 Phylogenetic trees of gut phages based on large subunit terminases. a Terminase_1; b Terminase_3; uncultured, black; Myoviridae, green; Siphoviridae, red; Podoviridae, blue. Unclassified cultured, yellow. Maximum-likelihood trees of all identified large subunit terminases from the identified large phage scaffolds and ENA phage genomes. The red branches in the trees denote the sequences from ENA phage genomes

membranes. The prevalence of this gene suggests that it may play an important role in gut microbiota and possibly serve as a marker for human gut phages. Another interesting accessory gene annotated as hemolysin (45\% identity to WP_044154808.1) was observed in a large phage scaffold (SRR341696|Scaffold32710_3, $179 \mathrm{~kb}$ ) that appeared in 16 WCMS samples. Hemolysin is suggested to cause lysis of red blood cells by destroying their cell membranes. The genes encoded by the phage scaffolds were compared against the VFDB with a cutoff of 1e-05, and 411 scaffolds had at least one hit to various virulence genes (Additional file 3: Table S3), which is suggestive of a gut pathogenic gene pool carried by phages.
Phage-bacteria partnership indicates phage specificity to their bacterial hosts

Based on the CRISPR-targeting method described by Stern et al. (Fig. 1a, Strategy I) [18], we paired the large phage scaffolds to their putative bacterial hosts (Fig. 4). In the 145 WCMS samples, we identified 1037 spacer sequences from 322 large bacterial scaffolds. These large bacterial scaffolds were generated from 112 metagenomic samples. We taxonomically assigned the large bacterial scaffolds as described in the "Methods" section, and 224 $(69.6 \%)$ of them were unambiguously assigned on the genus level. The most abundant genera were Bacteroides $(n=47)$, Eubacterium $(n=47)$, Megamonas $(n=45)$,

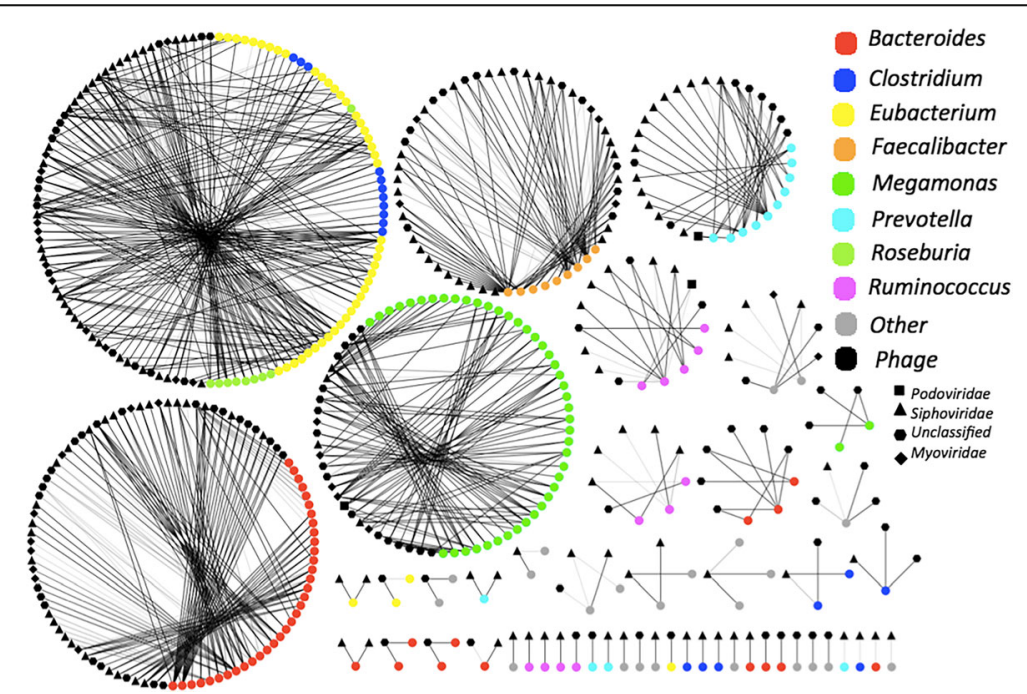

Fig. 4 The phage-bacterium specificity networks between the identified gut phages and the inferred hosts. Only large scaffolds ( $\geq 10 \mathrm{~kb}$ ) were considered. Spacers were extracted from CRISPR arrays and used to probe the corresponding phage scaffolds. Both phage and bacteria scaffolds were taxonomically assigned to the genus level. The networks were visualized by Cytoscape software. The width of the edges is proportional to the number of spacers of a bacterial scaffold paired to a phage scaffold. Only the bacterial scaffolds that could be assigned taxonomically were included 
Clostridium $(n=18)$, Prevotella $(n=14)$, Ruminococcus ( $n$ $=12)$, Faecalibacterium $(n=9)$, and Roseburia $(n=9)$.

Subsequently, the obtained spacer sequences were mapped to the large phage scaffolds. Only 10 (0.96\%) spacers and the paired phage scaffolds co-existed in the same WCMS samples (Fig. 4, Additional file 3: Table S4), suggesting that the spacers carried by CRISPRs provide the whole gut bacterial community highly effective protection from being infected by the corresponding phages. As shown in Fig. 4, the large phage scaffolds showed high specificity to the inferred hosts. Most $(98 \%, 314 / 321)$ of the phage scaffolds were paired to bacterial hosts within the same genera, while 7 phage scaffolds were paired to bacterial scaffolds from two genera (Eubacterium-Roseburia $(n=4)$, Eubacterium-Clostridium $(n=2)$, Coprococcus-Lachnospira $(n=1)$ ) (Fig. 4, Additional file 3: Table S4). However, these genera all belong to the order Clostridiales. Although wrong pairs due to short spacers cannot be excluded completely, this case implies that some gut phages are capable of infecting hosts across the genera in the order Clostridiales. Bacterial scaffolds of Bacteroides had the highest number $(25 \%, 77 / 308)$ of the paired phage scaffolds. These Bacteroides phages encode many functional accessory genes, such as anaerobic ribonucleosidetriphosphate reductase, implying the importance of $\mathrm{Bac}$ teroides phages in the gut ecosystem (Additional file 2: Figure S6). In conclusion, this network analysis reveals a global pattern of gut phage specificity to their bacterial hosts in the human gut environment.

The spacers in CRISPR arrays record the history of phages infecting bacterial hosts. We observed that $65 \%$ ( $n=275)$ of the 421 phage scaffolds were linked to 2-38 bacterial scaffolds and $72 \%(n=239)$ of the 321 bacterial scaffolds were "attacked" by multiple phage scaffolds $(n \geq 2)$ (Fig. 4, Additional file 3: Table S4). As shown in Fig. 4, most bacterial genera can be infected by phages from all three families in the order Caudovirales. A number of bacterial scaffolds harbor CRISPR arrays with multiple spacers matching to the same phage scaffolds (Fig. 4, Additional file 3: Table S4). These observations demonstrated a close and co-evolutionary interaction between phages and bacteria existing in the human gut.

\section{Significant alterations of the gut phageome in T2D were observed and validated}

A previous study indicated that the gut bacteriome is associated with T2D [3]. Considering the predator-prey relationship between the gut phages and bacteria, we expected to observe a reciprocal interaction between the phageome and bacteriome in the control and T2D samples. We first profiled the gut phageome in the human gut samples by defining pOTUs. The relative number for each pOTU in the samples was calculated according to the protocol described in the "Methods" section. Interestingly, we found that the relative numbers of the Myoviridae, Podoviridae, Siphoviridae, and unclassified_Caudovirales families increased significantly $(p<0.05$, FDR $<0.25$, Wilcoxon test) in the T2D samples (Fig. 5a). The phage alterations were further traced to the lowest-level taxon. We defined the core pOTUs $(n=58)$ as those that existed in more than $2 / 3(n>96)$ of the samples. Of these, 7 core pOTUs (4 Siphoviridae, 2 Podoviridae, and 1 unclassified family) were significantly different $(p<0.05$, FDR $<0.25)$ between the T2D and control groups. Interestingly, all increased in terms of the relative number in the T2D group (Fig. 5a). The inferred bacterial hosts of these pOTUs were putatively from the bacterial genera Enterobacteria, Escherichia, Lactobacillus, Pseudomonas, and Staphylococcus. We reanalyzed the overall different bacterial taxa between the T2D and control samples using MetaPhlAn and LEfSe (LDA score > 3) (Fig. 5b and Additional file 2: Figure S7), showing that the alterations of the gut phageome do not agree with the changes in the putative bacterial hosts.

To validate the significant alterations, we analyzed the 7 pOTUs in a separate stage II dataset with a larger sample size, containing 225 T2D $(n=116)$ and control $(n=$ 109) samples from Chinese adult individuals. We verified that all 7 pOTUs were significantly correlated $(p<$ 0.05 and FDR $<0.25$ ) with T2D (Additional file 3: Table S5). In this independent dataset, more pOTUs were associated with $\mathrm{T} 2 \mathrm{D}$, and the relative numbers of these pOTUs increased as well (Additional file 3: Table S5).

We also verified the significant increase in the number of phages in the T2D samples on the large phage scaffold level. The total number of reads mapped to the identified large phage scaffolds was counted and further normalized by the total read number of the samples. A Wilcoxon rank-sum test was performed to identify the difference in the normalized phage numbers between T2D and control samples. Our analysis showed a significant increase in the normalized phage number in the T2D samples (Wilcoxon test, $p=8.505 \mathrm{e}-11$, Fig. 5c).

\section{Co-occurrence/exclusion analysis uncovers a complex interaction between the gut phageome and the bacteriome}

The predator-prey relationship between phages and bacteria in the gut is central to gut microbiome equilibrium, but the global interactions between phages and bacteria have not yet been well defined. To determine the correlation between the core phages and bacteria (the pOTUs and bacterial genera detected in more than 72.5 samples (50\% of 145$)$ ), the network between pOTUs and bacterial genera was calculated by SparCC and visualized by Cytoscape [40, 46]. As shown in Fig. 6, in the resulting network of significant $(p<0.01)$ phage-bacteria interactions, each node is either a bacterial genera (hexagon) or pOTU (round). The network has 46 nodes, including 8 


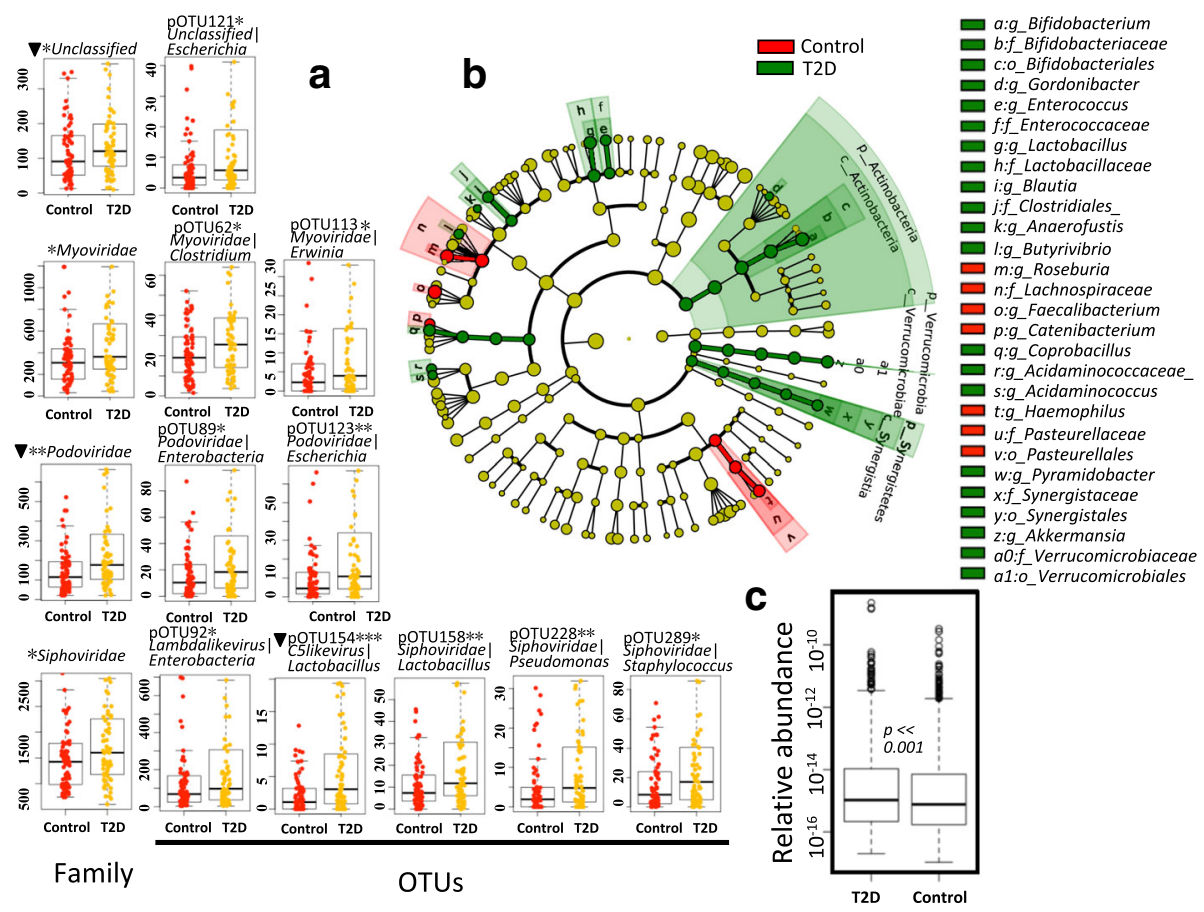

Fig. 5 Alterations of the phage and bacterial taxa in the T2D group and control group. a The pOTUs whose relative numbers increased in T2D samples. ${ }^{*}<0.05,{ }^{* *}<0.01,{ }^{* * *}<0.001$, FDR $<0.25$, triangle: FDR $<0.05$; Wilcoxon rank-sum test corrected by the Benjamini and Hochberg method. Only the highly prevalent pOTUs detected in $70 \%$ of the samples were included. $\mathbf{b}$ Alterations of bacterial taxa identified by LEfSe. Each circle's diameter is proportional to the taxon's abundance. Differences are represented in the color of the most abundant class. $\mathbf{c}$ The significant variation of the abundances of the identified phage scaffolds between the T2D group and control group; $p$ value $=8.505 \mathrm{e}-11$. In each WCMS sample, the phage abundance was calculated by normalizing all read numbers mapped to the identified large phage scaffolds against the total read number of the WCMS sample

bacterial and 38 phage nodes. The genera Escherichia (B24) and Bacteroides (B04) have the highest numbers of phage interactions. Escherichia interacts with 25 pOTUs, including 9 exclusions and 16 co-occurrences. Bacteroides has 6 exclusion interactions and 3 co-occurrence interactions. The genus Faecalibacterium (B17) has a co-occurrence interaction with the genus Roseburia (B15), and these two genera are enriched in the control group (Fig. 5b and Additional file 2: Figure S7). Faecalibacterium co-occurs with the pOTUs Podoviridae|Sinorhizobium (V35), Siphoviridae|Lactococcus (V53), and Siphoviridae|Skunalikevirus|Lactococcus (V75), and interestingly, these three pOTUs have co-occurrence interactions with more than two bacterial genera. Of the three pOTUs (unclassified|Escherichia (V83), Podoviridae|Enterobacteria (V32), and Podoviridae|Escherichia (V33)) enriched in the T2D samples (Fig. 5b and Additional file 2: Figure S7), all have co-occurrence interactions with Escherichia. In contrast, V33 and V32 have an exclusion interaction with Faecalibacterium and Eubacterium (B12), respectively. The bacterial hosts of pOTUs with co-occurrence interactions with Escherichia are putatively from Enterobacteria. Escherichia and Bacteroides significantly interact with the highest number of pOTUs, suggesting that they are the key microbes dominating the intestine ecosystem.

\section{Discussion}

This study used multiple sophisticated bioinformatic methods to characterize the phageome in fecal samples from T2D and normal Chinese adult individuals. To date, it is the largest study to correlate the gut phageome with T2D, and it substantially expands our understanding of the human gut phageome. More importantly, we demonstrated the significant T2D-specific alterations of the gut phageome. As the primary significance of this study, by large-scale comprehensive bioinformatic analyses, we identified 7 pOTUs associated with T2D patients and illustrated the comprehensive relationships between phages and their bacterial hosts in the gut microbiome.

WCMS data provide more information about phagebacteria relationships in the gut ecosystem than VLPderived metagenomic data and thus are suitable for phage-wide association studies. Based on the known phage genomes in the ENADB, we profiled the gut phageome in the WCMS data and VLP-based metagenomic data, resulting in a similar pattern of phageome distribution among the gut samples (Additional file 2: Figure 


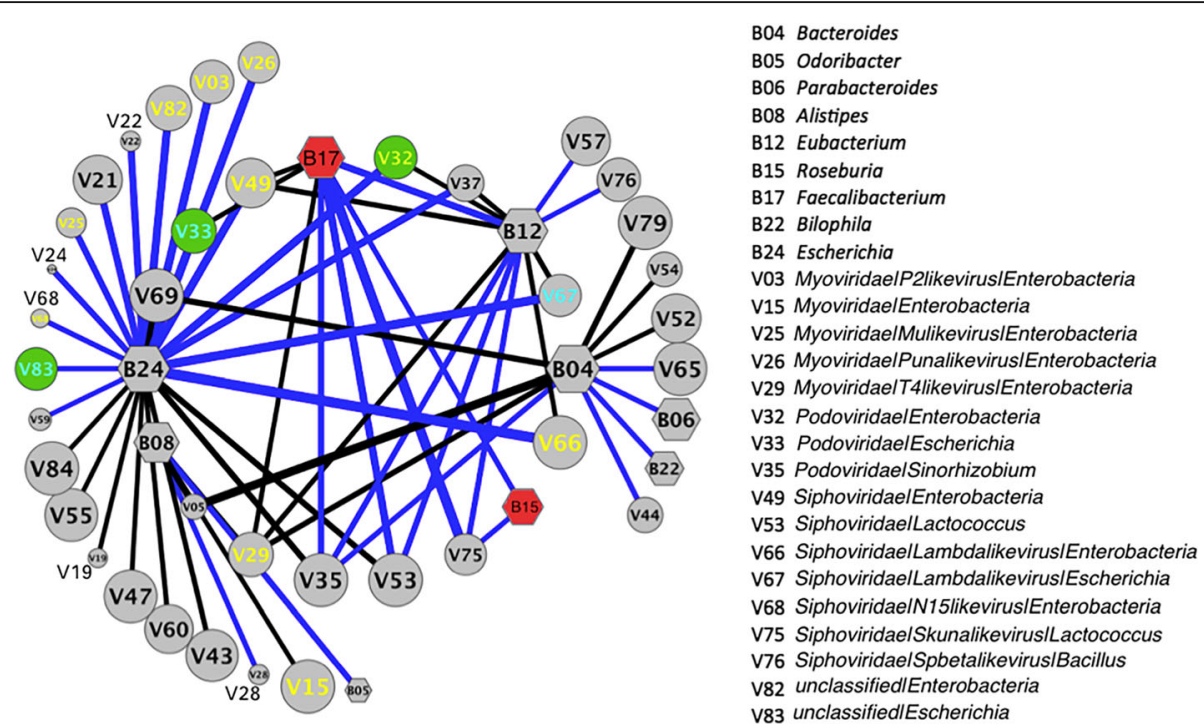

Fig. 6 The interaction network of bacterial genera and phage OTUs determined by SparCC. Nodes represent OTUs; round: pOTUs; hexagon: bacterial genera. Only bacterial genera and pOTUs with a high frequency (detected in more than 72.5 samples (50\%)) were considered. The co-correlation/exclusion interactions between bacterial genera and pOTUs as well as between bacterial genera were calculated with SparCC. The size of each node is proportional to the number of samples. The width of the edges between nodes is proportional to the correlation values between the nodes, with blue and black indicating the positive and negative correlations, respectively. Only edges corresponding to correlations greater than 0.3 and a p value less than 0.01 are shown, and unconnected nodes were omitted. The network layout was calculated by the Cytoscape software using a circular layout. The names of pOTUs with a putative Enterobacteria host are marked as yellow, and those with a putative Escherichia host are marked as cyan; the nodes representing the bacterial genera enriched in the control group are filled in with red, while the nodes representing the pOTUs enriched in the T2D group are filled in with green. The detailed names of the bacterial genera and only the pOTUs marked with colors were shown on the left panel of the figure. The detailed names of other pOTUs were shown in Additional file 3, Table S6
S1). This suggests that the WCMS and VLP data are equally suitable for gut phage detection. However, because of the lack of phage reference genomes and the high complexity of WCMS data, it is challenging to distinguish between the phage and the bacterial sequences in WCMS data. In this study, we used three strategies to overcome the difficulties in recovering large phage scaffolds from the WCMS data. As shown in Fig. 2c, the largest majority of the identified phage scaffolds were assigned taxonomically to the order of Caudovirales. Given the important function of terminase in phage DNA packaging of caudoviruses, the gene of terminase can be found on the genomes of various caudoviruses, and thus LST has been widely used as the marker gene for phylogenetic analysis of caudoviruses. To the best of our knowledge, we included the largest number of LSTs extracted from gut samples and all known phage genomes to determine the gut phage phylogeny (Fig. 3 and Additional file 2: Figure S4). This analysis expanded the diversity of gut phages and defined new lineages, implying that the viral studies dependent on the known phages might result in bias of insight into gut phageome.

Most of the gut phages described in this study are novel and are therefore undetected by reference-based methods. Analyses and characterization of these novel phage sequences demonstrated the number of accessory genes carried by the phage scaffolds. This expands our understanding of phage functions in the human gut microbiome. Failure to detect ssDNA phages can be considered as a limitation of the WCMS-based method in the phage detection. We did not intend to distinguish between phage and prophage sequences. Because of the dominance of temperate phages in the human gut ecosystem reported in previous studies, we believe that this study provides more insights into the human gut phageome than VLP-based studies, in terms of detecting both free phages and prophages [12, 14, 47].

Taking advantage of the WCMS data containing valuable information about phages and their hosts, we then paired phage scaffolds with bacterial scaffolds based on the CRISPR-targeting method described by Stern et al. [18]. We first showed in silico a global picture of phagehost specificities in the gut ecosystem via large-scale taxonomic assignment of each pair of scaffolds. As shown in Fig. 4, it can be concluded in silico that most of the gut phages have a narrow bacterial host range within the same genera. A tiny number of phages could cross-infect different bacterial genera (within Clostridiales). However, this finding is likely not in conflict with the highly specific nature of phages observed 
experimentally. The promiscuous phage is capable of evolving rapidly to infect new hosts by recruiting or mutating a new tail fiber gene.

Interactions between bacterial phages and their hosts dominate the gut ecosystem. Therefore, it is critical to identify the relationships between phages and bacteria to understand the potential role of phages in the development of T2D. However, only some of the phage scaffolds could be paired with the bacterial scaffolds based on the CRISPR-targeting method. Moreover, by mapping the reads across 145 samples to the identified phage scaffolds, we observed that most of the identified phage scaffolds exhibited high breadth of coverage and depth of coverage only in the samples where they were detected (Additional file 2: Figure S4a and b), suggesting that the gut phageomes are highly individualized, as described previously [10,12]. Although we observed a significant increase in the number of phages in T2D samples based on the identified phage scaffolds (Fig. 5c), alterations of the phageome and bacteriome could not be explained by some particular phage taxa. Thus, we tentatively defined pOTUs to determine the association between phages and bacteria. We identified 7 pOTUs that were significantly increased in the T2D group. Additionally, we estimated the richness of the gut phageome in T2D and control samples but did not find significant differences in the richness of the gut phageome between the two groups (Additional file 2: Figure S8). Therefore, given that temperate phages are dominant in the gut ecosystem $[10,13]$, we hypothesize that T2D-related factors enriched in the gut of T2D patients cause lysogenic phages to switch to the lytic cycle. Further investigations are required to determine the possible mechanisms underlying.

It is of interest to note that we found that the gut phages' pattern with regard to T2D is not mirrored in bacteria. According to the hypothesis of "Kill the Winner," phages are well known to be main predators of gut bacteria [48]. However, many recent studies suggested that bacteriophages play roles in intestinal physiology that are far more important than the alteration of bacterial communities by their infection. Norman et al. reported inflammatory bowel disease-specific alterations in the enteric virome [14]. Phages residing in mucosal surfaces can provide non-host-derived immunity against bacterial infections [26]. A growing number of evidences showed that phages are able to directly affect human $[14,19,20,25-27]$. For example, phages can act as antigens to stimulate host immunity and inflammation [49]. Here, we also found that the gut bacterial phages carried accessory genes responsible for human pathogenesis or human fitness (Additional file 2: Figure S6, Additional file 3: Table S3). In the light of this, it is not necessary to be surprising that the patterns of the phageome and bacteriome were distinct from each other
(Fig. 5), and that extensive co-occurences/exclusions between phages and bacteria existed in gut ecosystem (Fig. 6). Gut microbiota is a great complex community, in which, the commensal, mutualistic, and symbiotic relationships between different bacterial taxa, phages and human, phages and bacteria, bacteria and human, etc., might be established. Particularly, the genus of Bacteroides is the most abundant bacteria in the gut, and thus interacts with other members extensively. Figure 6 shows that many phages are associated with the genus of Bacteroides, likely reflecting indirect associations between Bacteriodes and other phages. Thus, our study implies a new direction to revisit the T2D risk. And we may have to take virome's impacts on human gut microbiome into consideration when we attempt to develop therapeutic approaches against $\mathrm{T} 2 \mathrm{D}$ via manipulating gut microbiome.

\section{Conclusions}

Here, for the first time, we correlated the gut phageome with T2D. Instead of a reciprocal relationship of phage specific to its bacterial host, a complex core interaction among bacteria and phages was revealed by a cooccurrence/exclusion analysis of bacterial genera and pOTUs. The T2D-associated changes in the phageome cannot simply be explained as co-variation with their altered bacterial hosts. These results imply that in addition to the cardinal and highly specific bacteria-phage interaction, other mechanism(s) may govern the phageome in the gut ecosystem. This study suggests that we should pay more attention to the role of phages in human microbiome studies and indicates the potential of gut phages in diagnostic and therapeutic applications.

\section{Additional files}

Additional file 1: This pdf file contains supplementary Material and Methods for the detailed descriptions of identification of the large phage scaffolds and definition of the pOTUs. (DOCX $101 \mathrm{~kb}$ )

Additional file 2: This pdf file contains the following supplementary figures: S1-S8. Legends for these figures are presented at the beginning of Additional file 1. (DOCX $3421 \mathrm{~kb}$ )

Additional file 3: This $x \mid s x$ file contains the following supplementary spreadsheets, each included as a separate tab in a single Microsoft Excel file: Tables2-TableS5. (XLSX 242 kb)

\section{Abbreviations}

BGR: Bacterial genus ratio; BPGDB: Bacterial and Phage Gene Database; ENA: European Nucleotide Archives; ENADB: European Nucleotide Archives genome database; ENAPDB: European Nucleotide Archives protein database; EPSGDB: Expanded Phage-Specific Gene database; IBD: Inflammatory bowel disease; LST: Large subunit terminase; NGS: Next-generation sequencing; PFR: Phage family ratio; pOTU: Phage operational taxonomic unit; SDB: Spacer DataBase (SDB); T2D: Type 2 diabetes; VLP: Virus-like particles; WCMS: Whole-community metagenomic sequencing 


\section{Acknowledgements}

We thank Dr. Zhiheng Pei from New York University, School of Medicine, who gave comments and suggestions.

\section{Funding}

This study is financially supported by Major Research Plan of the National Natural Science Foundation (NSFC) of China (91731302), the NSFC grants (31570115 and 31471270), Science and Technology Key Projects of Shenzhen (JSGG20160229145252927, JCYJ20160122143446357 and JCYJ20160229201759414), Strategic Priority Research Program of the Chinese Academy of Sciences (XDPB0305), 973 Program Grant (2014CB745202), and the Shenzhen Peacock Team Project (KQTD2015033117210153, KQTD2016112915000294).

\section{Availability of data and materials}

All WCMS reads used in this study were obtained from the NCBI short read archive (SRA) database (SRA045646) generated from a previously published Chinese T2D microbiome study [3]. Additionally, the corresponding assembled scaffolds generated from the exploration stage were downloaded from GigaDB (gigadb.org/dataset/100036) [3]. The VLP-based metagenomic sequencing datasets generated in three previous studies were downloaded from the EMBL-EBI (PRJEB7772) and NCBI SRA (SRP021107, SRX020505, and SRX020504) $[10,12,14]$. All datasets used in this study are listed in Additional file 1: Table S1.

\section{Authors' contributions}

YFM, XYY, and CLL conceived and designed the study. GQM performed the annotation of the Bacteroides-specific large phage scaffolds and created the figure. YFM performed all the analyses and created all the other figures. YFM, $X Y Y$, and CLL wrote a first draft of the paper, and $\Pi T$ contributed to the revisions. All authors read and approved the final manuscript.

\section{Ethics approval and consent to participate}

No ethics approval was required for this work.

\section{Consent for publication}

Not applicable.

\section{Competing interests}

The authors declare that they have no competing interests.

\section{Publisher's Note}

Springer Nature remains neutral with regard to jurisdictional claims in published maps and institutional affiliations.

Received: 9 February 2017 Accepted: 19 January 2018

Published online: 01 February 2018

\section{References}

1. Zhang C, Zhang M, Wang S, Han R, Cao Y, Hua W, Mao Y, Zhang X, Pang X, Wei C, Zhao G, Chen Y, Zhao L. Interactions between gut microbiota, host genetics and diet relevant to development of metabolic syndromes in mice. ISME J. 2010;4(2):232-41.

2. Bajzer M, Seeley RJ. Physiology: obesity and gut flora. Nature. 2006; 444(7122):1009-10.

3. Qin J, Li Y, Cai Z, Li S, Zhu J, Zhang F, Liang S, Zhang W, Guan Y, Shen D, Peng Y, Zhang D, Jie Z, Wu W, Qin Y, Xue W, Li J, Han L, Lu D, Wu P, Dai Y, Sun X, Li Z, Tang A, Zhong S, Li X, Chen W, Xu R, Wang M, Feng Q, Gong M, Yu J, Zhang Y, Zhang M, Hansen T, Sanchez G, Raes J, Falony G, Okuda S, Almeida M, LeChatelier E, Renault P, Pons N, Batto JM, Zhang Z, Chen H, Yang R, Zheng W, Li S, Yang H, Wang J, Ehrlich SD, Nielsen R, Pedersen O, Kristiansen K, Wang J. A metagenome-wide association study of gut microbiota in type 2 diabetes. Nature. 2012;490(7418):55-60.

4. Yano JM, Yu K, Donaldson GP, Shastri GG, Ann P, Ma L, Nagler CR, Ismagilov RF, Mazmanian SK, Hsiao EY. Indigenous bacteria from the gut microbiota regulate host serotonin biosynthesis. Cell. 2015;161(2):264-76.

5. Cox LM, Yamanishi S, Sohn J, Alekseyenko AV, Leung JM, Cho I, Kim SG, Li H, Gao Z, Mahana D, Zarate Rodriguez JG, Rogers AB, Robine N, Loke P, Blaser MJ. Altering the intestinal microbiota during a critical developmental window has lasting metabolic consequences. Cell. 2014;158(4):705-21.
6. Cox LM, Blaser MJ. Antibiotics in early life and obesity. Nat Rev Endocrinol 2015;11(3):182-90.

7. Manrique P, Bolduc B, Walk ST, van der Oost J, de Vos WM, Young MJ. Healthy human gut phageome. Proc Natl Acad Sci U S A. 2016:

8. Wylie KM, Weinstock GM, Storch GA. Emerging view of the human virome. Transl Res. 2012;160(4):283-90.

9. Paez-Espino D, Eloe-Fadrosh EA, Pavlopoulos GA, Thomas AD, Huntemann M, Mikhailova N, Rubin E, Ivanova NN, Kyrpides NC. Uncovering earth's virome. Nature. 2016;536(7617):425-30.

10. Minot S, Sinha R, Chen J, Li H, Keilbaugh SA, Wu GD, Lewis JD, Bushman FD. The human gut virome: inter-individual variation and dynamic response to diet. Genome Res. 2011;21(10):1616-25.

11. Minot S, Grunberg S, Wu GD, Lewis JD, Bushman FD. Hypervariable loci in the human gut virome. Proc Natl Acad Sci U S A. 2012;109(10):3962-6.

12. Minot S, Bryson A, Chehoud C, Wu GD, Lewis JD, Bushman FD. Rapid evolution of the human gut virome. Proc Natl Acad Sci U S A. 2013;110(30):12450-5.

13. Reyes A, Haynes M, Hanson N, Angly FE, Heath AC, Rohwer F, Gordon J. Viruses in the faecal microbiota of monozygotic twins and their mothers. Nature. 2010;466(7304):334-8.

14. Norman JM, Handley SA, Baldridge MT, Droit L, Liu CY, Keller BC, Kambal A, Monaco CL, Zhao G, Fleshner P, Stappenbeck TS, McGovern DP, Keshavarzian A, Mutlu EA, Sauk J, Gevers D, Xavier RJ, Wang D, Parkes M, Virgin HW. Disease-specific alterations in the enteric virome in inflammatory bowel disease. Cell. 2015;160(3):447-60.

15. Qin J, Li R, Raes J, Arumugam M, Burgdorf KS, Manichanh C, Nielsen T, Pons N, Levenez F, Yamada T, Mende DR, Li J, Xu J, Li S, Li D, Cao J, Wang B, Liang $H$, Zheng H, Xie Y, Tap J, Lepage P, Bertalan M, Batto JM, Hansen T, Le Paslier D, Linneberg A, Nielsen HB, Pelletier E, Renault P, Sicheritz-Ponten T, Turner K, Zhu H, Yu C, Li S, Jian M, Zhou Y, Li Y, Zhang X, Li S, Qin N, Yang H, Wang J, Brunak S, Dore J, Guarner F, Kristiansen K, Pedersen O, Parkhill J, Weissenbach J, Meta HITC, Bork P, Ehrlich SD, Wang J. A human gut microbial gene catalogue established by metagenomic sequencing. Nature. 2010;464(7285):59-65.

16. Ogilvie LA, Bowler LD, Caplin J, Dedi C, Diston D, Cheek E, Taylor H, Ebdon JE, Jones BV. Genome signature-based dissection of human gut metagenomes to extract subliminal viral sequences. Nat Commun. 2013:4:2420

17. Waller AS, Yamada T, Kristensen DM, Kultima JR, Sunagawa S, Koonin EV, Bork P. Classification and quantification of bacteriophage taxa in human gut metagenomes. ISME J. 2014;8(7):1391-402.

18. Stern A, Mick E, Tirosh I, Sagy O, Sorek R. CRISPR targeting reveals a reservoir of common phages associated with the human gut microbiome. Genome Res. 2012;22(10):1985-94

19. Virgin HW. The virome in mammalian physiology and disease. Cell. 2014; 157(1):142-50.

20. Miedzybrodzki R, Switala-Jelen K, Fortuna W, Weber-Dabrowska B, Przerwa A, Lusiak-Szelachowska M, Dabrowska K, Kurzepa A, Boratynski J, Syper D, Pozniak G, Lugowski C, Gorski A. Bacteriophage preparation inhibition of reactive oxygen species generation by endotoxin-stimulated polymorphonuclear leukocytes. Virus Res. 2008;131(2):233-42.

21. Przerwa A, Zimecki M, Switala-Jelen K, Dabrowska K, Krawczyk E, Luczak M, Weber-Dabrowska B, Syper D, Miedzybrodzki R, Gorski A. Effects of bacteriophages on free radical production and phagocytic functions. Med Microbiol Immunol. 2006;195(3):143-50.

22. Ksendzovsky A, Walbridge S, Saunders RC, Asthagiri AR, Heiss JD, Lonser RR. Convection-enhanced delivery of $\mathrm{m} 13$ bacteriophage to the brain. J Neurosurg. 2012;117(2):197-203.

23. Dabrowska K, Opolski A, Wietrzyk J, Switala-Jelen K, Godlewska J, Boratynski J, Syper D, Weber-Dabrowska B, Gorski A. Anticancer activity of bacteriophage $\mathrm{t} 4$ and its mutant hap1 in mouse experimental tumour models. Anticancer Res. 2004;24(6):3991-5.

24. Eriksson F, Culp WD, Massey R, Egevad L, Garland D, Persson MA, Pisa P. Tumor specific phage particles promote tumor regression in a mouse melanoma model. Cancer Immunol Immunother. 2007;56(5):677-87.

25. Pajtasz-Piasecka E, Rossowska J, Dus D, Weber-Dabrowska B, Zablocka A, Gorski A. Bacteriophages support anti-tumor response initiated by dc-based vaccine against murine transplantable colon carcinoma. Immunol Lett. 2008; 116(1):24-32.

26. Barr JJ, Auro R, Furlan M, Whiteson KL, Erb ML, Pogliano J, Stotland A Wolkowicz R, Cutting AS, Doran KS, Salamon P, Youle M, Rohwer F. Bacteriophage adhering to mucus provide a non-host-derived immunity. Proc Natl Acad Sci U S A. 2013;110(26):10771-6. 
27. Ly M, Abeles SR, Boehm TK, Robles-Sikisaka R, Naidu M, Santiago-Rodriguez T, Pride DT. Altered oral viral ecology in association with periodontal disease. MBio. 2014;5(3):e01133-14.

28. Li R, Yu C, Li Y, Lam TW, Yiu SM, Kristiansen K, Wang J. SOAP2: an improved ultrafast tool for short read alignment. Bioinformatics. 2009;25(15):1966-7.

29. Lonc E. Principles and use of numerical taxonomy in the classification of arthropods. Wiad Parazytol. 1985;31(4-6):515-8.

30. Kristensen DM, Waller AS, Yamada T, Bork P, Mushegian AR, Koonin EV. Orthologous gene clusters and taxon signature genes for viruses of prokaryotes. J Bacteriol. 2013;195(5):941-50.

31. Segata N, Waldron L, Ballarini A, Narasimhan V, Jousson O, Huttenhower C. Metagenomic microbial community profiling using unique clade-specific marker genes. Nat Methods. 2012;9(8):811-4.

32. Zhu W, Lomsadze A, Borodovsky M. Ab initio gene identification in metagenomic sequences. Nucleic Acids Res. 2010;38(12):e132.

33. Tatusov RL, Fedorova ND, Jackson JD, Jacobs AR, Kiryutin B, Koonin EV, Krylov DM, Mazumder R, Mekhedov SL, Nikolskaya AN, Rao BS, Smirnov S, Sverdlov AV, Vasudevan S, Wolf YI, Yin JJ, Natale DA. The cog database: an updated version includes eukaryotes. BMC Bioinformatics. 2003;4:41.

34. Davidsen T, Beck E, Ganapathy A, Montgomery R, Zafar N, Yang Q, Madupu R, Goetz P, Galinsky K, White O, Sutton G. The comprehensive microbial resource. Nucleic Acids Res. 2010;38(Database issue):D340-5.

35. Sonnhammer EL, Eddy SR, Birney E, Bateman A, Durbin R. Pfam: multiple sequence alignments and hmm-profiles of protein domains. Nucleic Acids Res. 1998;26(1):320-2.

36. Marchler-Bauer A, Panchenko AR, Shoemaker BA, Thiessen PA, Geer LY, Bryant SH. CDD: a database of conserved domain alignments with links to domain three-dimensional structure. Nucleic Acids Res. 2002;30(1):281-3.

37. Chen L, Xiong Z, Sun L, Yang J, Jin Q. VFDB 2012 update: toward the genetic diversity and molecular evolution of bacterial virulence factors. Nucleic Acids Res. 2012;40(Database issue):D641-5.

38. Finn RD, Coggill P, Eberhardt RY, Eddy SR, Mistry J, Mitchell AL, Potter SC, Punta M, Qureshi M, Sangrador-Vegas A, Salazar GA, Tate J, Bateman A. The Pfam protein families database: towards a more sustainable future. Nucleic Acids Res. 2016:44(D1):D279-85.

39. Tamura K, Stecher G, Peterson D, Filipski A, Kumar S. Mega6: molecular evolutionary genetics analysis version 6.0. Mol Biol Evol. 2013;30(12):2725-9.

40. Friedman J, Alm EJ. Inferring correlation networks from genomic survey data. PLoS Comput Biol. 2012;8(9):e1002687.

41. Demchak B, Hull T, Reich M, Liefeld T, Smoot M, Ideker T, Mesirov JP. Cytoscape: the network visualization tool for GenomeSpace workflows. F1000Res. 2014;3:151.

42. Segata N, Izard J, Waldron L, Gevers D, Miropolsky L, Garrett WS Huttenhower C. Metagenomic biomarker discovery and explanation. Genome Biol. 2011;12(6):R60.

43. Hu JX, Zhao H, Zhou HH. False discovery rate control with groups. J Am Stat Assoc. 2010;105(491):1215-27.

44. Dutilh BE, Cassman N, McNair K, Sanchez SE, Silva GG, Boling L, Barr J, Speth DR, Seguritan V, Aziz RK, Felts B, Dinsdale EA, Mokili JL, Edwards RA. A highly abundant bacteriophage discovered in the unknown sequences of human faecal metagenomes. Nat Commun. 2014;5:4498.

45. Li J, Jia H, Cai X, Zhong H, Feng Q, Sunagawa S, Arumugam M, Kultima JR, Prifti E, Nielsen T, Juncker AS, Manichanh C, Chen B, Zhang W, Levenez F, Wang J, Xu X, Xiao L, Liang S, Zhang D, Zhang Z, Chen W, Zhao H, Al-Aama $J$ Y, Edris S, Yang H, Wang J, Hansen T, Nielsen HB, Brunak S, Kristiansen K, Guarner F, Pedersen O, Dore J, Ehrlich SD, Meta HITC, Bork P, Wang J, Meta HITC. An integrated catalog of reference genes in the human gut microbiome. Nat Biotechnol. 2014;32(8):834-41.

46. Shannon P, Markiel A, Ozier O, Baliga NS, Wang JT, Ramage D, Amin N Schwikowski B, Ideker T. Cytoscape: a software environment for integrated models of biomolecular interaction networks. Genome Res. 2003;13(11):2498-504.

47. Cadwell K. Expanding the role of the virome: commensalism in the gut. J Virol. 2015;89(4):1951-3.

48. Winter C, Bouvier T, Weinbauer MG, Thingstad TF. Trade-offs between competition and defense specialists among unicellular planktonic organisms: the "killing the winner" hypothesis revisited. Microbiol Mol Biol Rev. 2010;74(1):42-57.

49. Eriksson F, Tsagozis P, Lundberg K, Parsa R, Mangsbo SM, Persson MA, Harris RA, Pisa P. Tumor-specific bacteriophages induce tumor destruction through activation of tumor-associated macrophages. J Immunol. 2009. 182(5):3105-11.

\section{Submit your next manuscript to BioMed Central and we will help you at every step:}

- We accept pre-submission inquiries

- Our selector tool helps you to find the most relevant journal

- We provide round the clock customer support

- Convenient online submission

- Thorough peer review

- Inclusion in PubMed and all major indexing services

- Maximum visibility for your research

Submit your manuscript at www.biomedcentral.com/submit
) Biomed Central 